\title{
EVALUATION OF ALVEOLAR OSTEOTOMY FOR CORRECTION OF VERTICAL BONE RESORPTION IN ANTERIOR MANDIBULAR RIDGE USING A MIXTURE OF TWO GRAFTED MATERIALS
}

\author{
Passent A. Younis ${ }^{1}$ B.sc, Ahmed M. Elsabbagh ${ }^{2}$ Phd, Riham M. Eldibany ${ }^{2}$ Phd.
}

\begin{abstract}
INTRODUCTION: Extensive loss of alveolar bone and teeth in the anterior mandible presents a complex problem for reconstruction. Alveolar osteotomy technique with the use of mixture of hydroxy apatite and beta-tricalcium phosphate together with injectable PRF is one of the reliable methods to restore vertical ridge deficiencies.

OBJECTIVES: : Evaluate vertical ridge augmentation using sandwich osteotomy technique in anterior atrophic mandible and the use of hydroxy apatite, beta-tri-calcium phosphate mixed with injectable PRF as a grafting material.

MATERIALS AND METHODS: This study was conducted clinically on 8 patients indicated for vertical ridge augmentation by sandwich technique osteotomy and fixation of the osteomatized segment by micro-plates and screws, and the placement of Ha- BTCP mixed with I-PRF as interpositional graft material. Patients were evaluated clinically and radiographically by using panoramic radiograph and cone beam computed tomography (CBCT).

RESULTS: In this study no severe pain was recorded postoperatively. No signs of inflammation or swelling were observed in almost all of the patients along the follow up period. Vertical bone height gain of the residual ridge was satisfactory and prepared the ridge to receive final prosthesis successfully.

CONCLUSIONS Sandwich osteotomy technique with Ha- BTCP mixed with I-prf is a reliable method to restore vertical ridge deficiencies in anterior mandible.

KEYWORDS: Sandwich technique, Hydroxy apatite- beta-tricalcium phosphate, Injectable-PRF, vertical ridge atrophy.
\end{abstract}

1- Instructor at Department of Oral and Maxillofacial Surgery, Faculty of Dentistry, Alexandria University.

2- Professor at Department of Oral and Maxillofacial Surgery, Faculty of Dentistry, Alexandria University.

\section{INTRODUCTION}

The extent of the residual ridge reduction (RRR) is especially marked in anterior parts of the maxillary and mandibular ridges. According to Atwood, continuous reduction in alveolar ridge is regarded as a 'major oral disease' including anatomical, metabolic, prosthetic and functional factors. A significantly reduced alveolar ridge creates a number of problems complicating prosthodontic rehabilitation and a construction of complete dentures (1).

The treatment of vertical alveolar defects is a most challenging condition in alveolar ridge augmentation. Previously many bone augmentation techniques have been developed and the debate was which of them would be preferable and implementable in such challenging situations (2).

Since its description in the 1970s, the sandwich technique has been found to be reliable for the reconstruction of atrophic mandibles (3). Alveolar sandwiching osteotomy is a relatively the alternating technique to distraction osteogenesis for vertical bone augmentation which has proved relatively safe, with successful long - term results of 5-10mm vertical bone augmentation (4).

The sandwich osteotomy allows for the positioning of the graft in a well- defined area as well as offering adequate blood supply to maintain new bone growth (5).

The rationale of the interpositional techniques is based on the theory that biomaterial placed between 2 pieces of pedicled bone with internal cancellous bone will undergo rapid and complete healing and graft incorporation with a lower percentage of resorption (5).

Although the use of autogenous bone has been widely accepted as the gold standard augmentation material; intraand extra-oral donor site morbidity, potential complications and risks associated with the harvesting procedures have been reported (6).

To overcome these problems synthetic bio-inert materials are currently used as an alternative to autogenous bone graft. Hydroxy apatite (HA) and Beta tri-calcium phosphate ( $\beta$-TCP), which belong to the calcium phosphate ceramics group, are biocompatible and osteo-conductive (7).

The use of PRF in conjunction with grafting materials would accelerate bone regeneration. Recent studies have shown good results using PRF in order to promote bone regeneration. Nilüfer Bölükbaşı et al in 2013 (8) concluded that PRF in addition to BCP may favor the formation of new bone. PRF has a new injectable form Injectable PRF (IPRF).

Thus the aim of this study was to evaluate clinically and radiographically the alveolar sandwich osteotomy technique with the use of Biphasic calcium phosphate mixed with injected platelets rich fibrin (IPRF) as an interpositional augmented material to increase vertical bone height in anterior mandibular alveolar ridge resorption.

\section{MATERIALS AND METHODS}

\section{Ethical consideration}

Benefits and drawbacks were emphasized for each patient enrolled in this study, then an informed written consent was signed by each patient to approve the procedure. This study was approved by Faculty of Dentistry Ethics committee.

\section{Study design}

This study was conducted on eight patients with anterior mandibular vertical bone resorption attending the outpatient clinic of the Oral and Maxillofacial Surgery Department, and the removable Prosthodontic Department at the Faculty of Dentistry, Alexandria University. 


\section{Inclusion criteria}

Patients with resorbed anterior mandibular alveolar ridge in the vertical dimension who find difficulty in prosthetic rehabilitation, and require from 5 to $10 \mathrm{~mm}$ increase in the vertical height. Patients were selected from both sexes with age ranged from 40 to 65 years Patients should be systemically healthy and psychologically accepting the involved procedures.

\section{Exclusion criteria}

Patients with mandibular alveolar ridge height less than $10 \mathrm{~mm}$.Patients with inadequate oral hygiene, with para functional habits or with any habits that might reduce the blood flow and retard healing such as heavy smokers and alcoholism were excluded.

\section{Materials}

Synthetic Hydroxyapatite and Beta tricalcium phosphate (DM bone, Meta Biomed $\AA$, Osong-eup, Korea) as a graft material. Its a bioactive resorbable bone graft material. It is a ceramic constituted of two-phases of silicon ( $<1 \mathrm{wt} \%)$ containing coralline $60 \%$ hydroxyapatite and $40 \%$ betatricalcium phosphate

Injectable PRF (IPRF ) which is a leukocytes and platelets concentrate containing many growth factors. It was prepared, by aspirating $10 \mathrm{ml}$ brachial venous blood and using the customized tube Falcon ${ }^{\circledR} 15 \mathrm{~mL}$ high-clarity polypropylene (PP) ( Attaka, Italy). The blood was centrifuged at a speed 3500 Revolution Per Minute (RPM) for 8 minutes and adding $0.2 \mathrm{ml}$ calcium chloride. The injectable PRF gives bone steak when mixed with the bone graft.

PRF membrane was prepared according to the protocol developed by Choukroun et al (9).After obtaining $20 \mathrm{ml}$ of brachial venous blood, the blood was divided into two $10 \mathrm{ml}$ sterile glass tubes without anti-coagulant. Immediate centrifugation was performed at 3000 RPM for 10 minutes using a table centrifuge.( Jiangsu Zhengji, China).

This process yields in each test tube an upper layer of serum, middle layer of PRF and a bottom layer of RBC's. The serum layer was discarded and the PRF layer was separated from the RBC's layer using scissors.

The PRF was then removed and gently inserted between two sterile glass slabs wrapped by gauze and gently pressed against each other giving the PRF membrane.

Titanium Jeil Medical cooperation micro-plates and screws (Seoul, Korea) were used, the micro-plates are 0.5 $\mathrm{mm}$ in thickness. T-shaped (5 holes) and double Y shaped (6 holes) micro-plates were used in this study. Eight microscrews of length $6 \mathrm{~mm}$ were used. The screws are threaded with diameter of thread $1.2 \mathrm{~mm}$ and diameter of screw head $2 \mathrm{~mm}$.

\section{Methods}

\section{Pre-surgical phase}

Medical and dental history was conducted on all selected patients and laboratory investigations were done to exclude any systemic disease that can interfere with the surgical procedure. Local visual examination and palpation of the entire oral and paraoral tissues were performed, and evaluation of the ridge to be augmented considering the contour, height and healthy normal condition of the overlying mucosa.

\section{Radiographic examination}

Panoramic radiographs were done for pre-operative preliminary assessment and Cone beam Computed tomography scans (CBCT) were done to measure the vertical height of bone from the alveolar crest till the inferior border of the mandible, and to determine bone density, also it was used for localization of the osteotomy lines. (Figure 1)

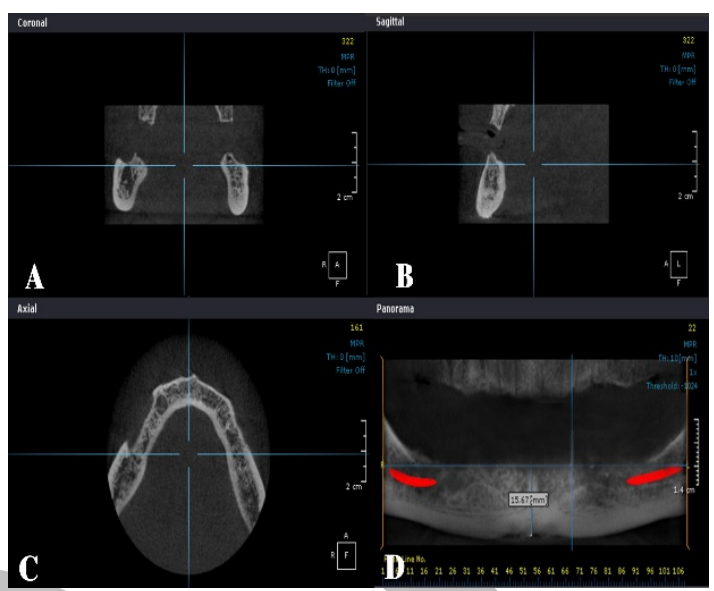

Figure 1: Preoperative CBCT showing atrophic anterior mandible.

Preoperative antibiotic was given to all patients in the form of Amoxicillin trihydrate $(875 \mathrm{mg}$ ) and clavulanic potassium (125 mg) (AugmentinTM 1 g, Glaxo Smith Kline, Englnd ); 1 capsule every 12 hours for 2 days.

\section{Surgical phase}

Patients were operated under general anesthesia with nasotracheal intubation. Disinfection of the patient's face, oral and para-oral area were done using povidone- iodine (Betadine, the Arab Drug CO., Cairo Egypt) and complete isolation of the working field was made using sterile towels.

Local Anaesthesia was injected at the site of incision to reduce bleeding using 2\% Mepivacaine HCL with 1: 20,000 Levonordefrin. (Scandonest 2\% L, Septodont, France) then a labial subcrestal horizontal incision was performed extending from the canine -canine region. The mucoperiosteal flap was reflected downwards using a periosteal elevator to expose the underlying bone. No mucoperiosteal dissection was performed towards the alveolar crest or on the lingual side to preserve an adequate blood supply to the segmented bone. (Figure 2)

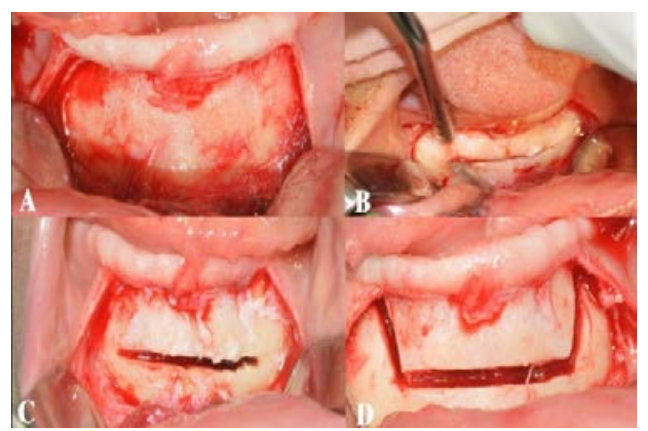

Figure 2: Surgical procedure: A: Flap reflected exposing the anterior mandibleB: Cutting into bone to perform the osteotomy lines using piezoelectric device. C: The horizontal osteotomy line. D: Completing the cutting process by performing the 2 vertical osteotomy lines. 
Using the piezoelectric SB PO20 insert, (Surgybone, Silfradent srl, Sofia, Italy) a horizontal osteotomy of the anterior mandibular alveolar ridge was accomplished extending from the canine - canine region, about 5-10 mm below the crest of the ridge, reaching the full depth of bone on the lingual side.

Another two slightly diverged vertical osteotomies were done on each end of the horizontal cut up till the alveolar crest. The osteotomy was completed by introducing a small osteotome through the osteotomy site, then with gentle wedging movement of the osteotome a free trapezoid - bone segment that remained attached to the lingual and crestal periosteum and mucosa was created. (Figure 2)

The segment was displaced $5 \mathrm{~mm}$ crestally which was measured by a disposable ruler. Then fixation of the segmented bone was done by two titanium micro-plates and four screws for each plate to obtain stability.

IPRF was prepared and mixed with the Ha- BCP particles to form the bone graft steak. The created gap was filled with the bone graft steak and covered by the PRF membrane. (Figure $3,4)$

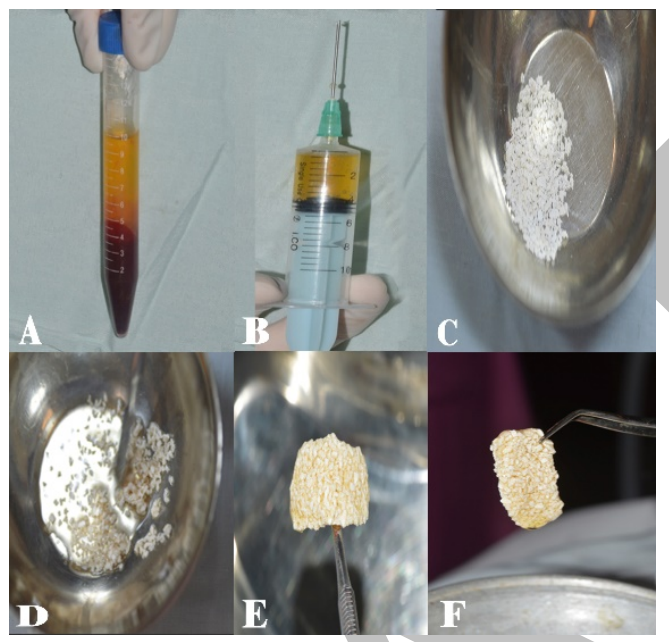

Figure 3: I-PRF preparation and its mixing with bone graft to form the bone graft steak.

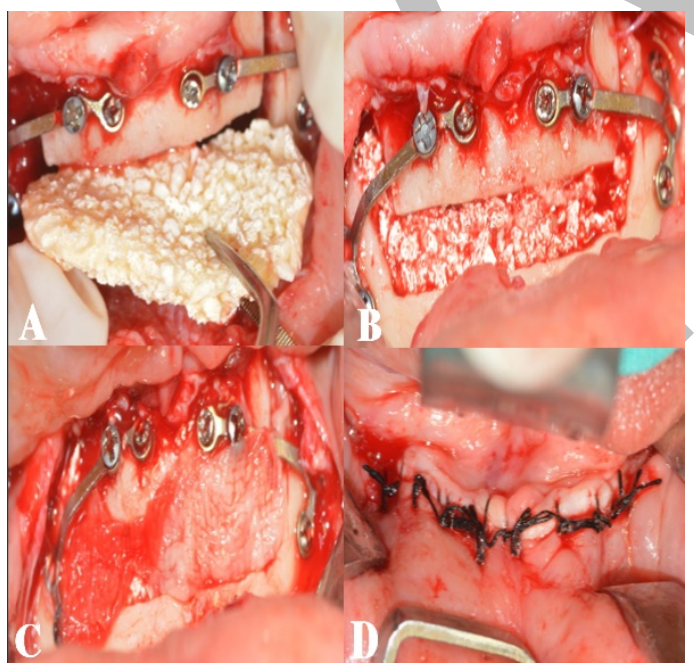

Figure 4: A: Placement of the graft material in the pre-created gap. B: Covering the graft with PRF membrane. C: Suturing with 000 black silk.
Slight undermining of the mucoperiosteal flap was done to allow stretching and repositioning of the subcrestal horizontal mucoperiosteal flap .Primary wound closure was achieved by $3 / 0$ black silk suture material with horizontal mattress sutures alternated with interrupted sutures. (Figure 4)

\section{Post-surgical phase}

A pressure pack was applied to the surgical site for about 30 minutes and post- operative instructions were given to the patients including cold packs on the chin area intermitting for two hours during the first day, patients were asked to avoid chewing hard food on the site of surgery, and no removable prosthesis was allowed for 4 months.

Amoxicillin trihydrate (875 $\mathrm{mg})$ and clavulanic potassium (125 mg) (AugmentinTM 1g, Glaxo Smith Kline) every 12 hours was continued postoperatively for 5 days, Ibuprofen (Nova profen, Sanofi Egypt) $400 \mathrm{mg} ; 1$ tablet every 8 hours after meals for 4 days as an non-steroidal antiinflammatory drug and $0.12 \%$ chlorohexidine (Hexitol, The Arab Drug CO., Cairo Egypt) warm mouth wash 3 times daily for the following 5 postoperative days.

Sutures were removed 8-10 days post surgically.

\section{Follow up phase}

All patients were examined the day after surgery, after one week, weekly for the first month and then after 4 months postoperatively for any signs of pain, swelling, infection, wound dehiscence or any other complications. Pain was evaluated by Visual Analogue Scale (VAS) (10) and swelling was evaluated by Tape measuring method for evaluation of facial edema where distance from the tragus on the right side to the tragus on the left side was measured using a thread.

Immediately post-operative (within the first week) CBCT was made and then after 4 months postoperatively to compare vertical ridge height and bone density changes using Hounsfield scale (HU) using BlueSkyBio 64-bit (BlueSkyBio , USA). After CBCT acquisition, the Dicom file of the block was exported and imported to BlueSkyBio 64-bit version software for bone density assessment. A Bone Density measurement icon was used at the region of interest to give a reading in a Hounsfield unit (6 points at each ROI).The mean bone density at the site of the grafted area was calculated by measuring the bone density at 12 points in the vicinity of the grafted area then taking their average to determine the mean bone density at 4 months postoperatively. (Figure 5)

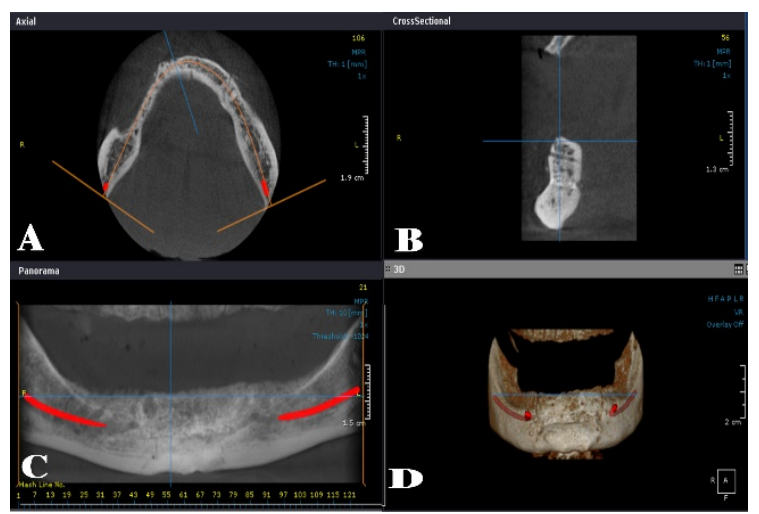

Figure 5: CBCT at 4 months postoperative showing the increased vertical height of anterior mandible. 
After the 4 months follow up period final rehabilitation of the augmented ridge was done successfully by construction of complete denture. (Figure 6)

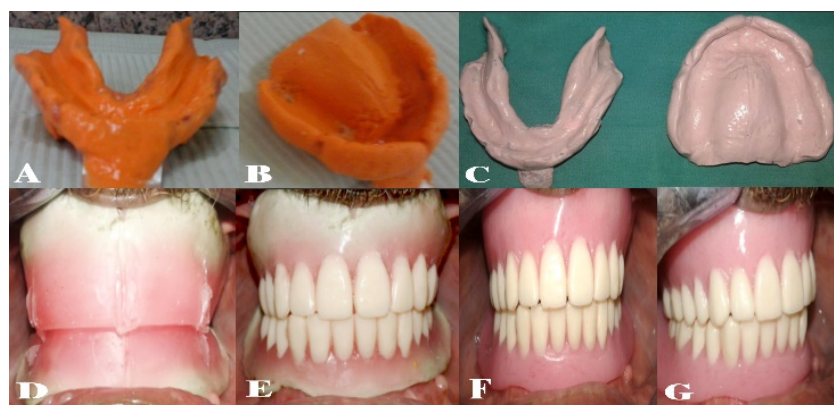

Figure 6: Complete denture fabrication as final prosthesis.

Appropriate statistical analysis was used to evaluate the outcomes. Means, standard deviations and median were calculated to describe data collected from the patients. ANOVA test with a Greenhouse-Geisser correction was used to evaluate the results. The level of significance was set at $\mathrm{F}(0.250,149.11)=4175.08, \mathrm{P}<0.005$.

\section{RESULTS}

In this study eight patients were selected, the selected patients were 5 males (62.5\%) and 3 females (37.5\%) and their age ranged from 55 to 62 years with a mean of $60.13 \pm 2.8$. Patients' ridge height ranged from $15.1 \mathrm{~mm}$ to $16.9 \mathrm{~mm}$, with a mean of $16.16 \pm 0.63 \mathrm{~mm}$ measured from preoperative CBCT.

They were evaluated post-operatively both clinically and radiographically.

\section{Clinical evaluation}

\section{Pain}

The average pain recorded during the first 3 days postoperatively was 7.75 and then declined to 6.25 in the fourth and fifth days reaching 1.5 at the end of the first postoperative week. Then the pain disappeared completely.

\section{Swelling}

Post-operative swelling has been developed in all patients with variable degrees. All patients showed slight swelling that was maximum the first 2 post-operative days then gradually subsides, until it completely disappeared at the end of the 1st post-operative week.

\section{Paresthesia}

Subjectively all patients had some transient postsurgical parethesia, that lasted for maximum 6 weeks postoperatively and recovered without any medications.

\section{Wound dehiscence}

Healing was uneventful in all cases with the absence of postoperative complications except for one case who exhibited wound dehiscence in some areas along the incision line and this case was treated by daily local irrigation with saline mixed with broad spectrum antibiotic cefotaxime $1 \mathrm{~g}$ vial (Cefotax, EPICO, Egypt)* on dental chair and at the same time the patient was instructed to maintain meticulous oral hygiene using Chlorhexidine and warm saline mouthwash at home. The dehiscence healed spontaneously 3 weeks later with the exfoliation of small bony spicule.

\section{Radiographic evaluation \\ Bone height evaluation}

The preoperative bone height ranged from $15.1 \mathrm{~mm}$ to $16.9 \mathrm{~mm}$, with a mean of $16.16 \pm 0.63 \mathrm{~mm}$.

Immediate post-operative CBCT was done to be compared with the preoperative CBCT to evaluate the vertical difference in height and it ranged from $5 \mathrm{~mm}$ to $5.6 \mathrm{~mm}$ with mean $5.3 \pm 0.2 \mathrm{~mm}$.

On the 4th post-operative month there was a statistically significant change in bone height to range from $20.3 \mathrm{~mm}$ to $22.2 \mathrm{~mm}$ with a mean of $(21.44 \pm 0.62 \mathrm{~mm})$ and a mean percentage change of $32.69 \%$. (Table 1 )

Table 1: Comparison between preoperative and postoperative bone height.

\begin{tabular}{|l|c|c|c|}
\hline & \multicolumn{3}{|c|}{ Bone height } \\
\cline { 2 - 4 } & Preoperative & $\begin{array}{c}\text { Delayed post- } \\
\text { operative }\end{array}$ & \% change \\
\hline Min.-Max. & $15.1-16.9 \mathrm{~mm}$ & $20.3-22.2 \mathrm{~mm}$ & $29.6-36.1$ \\
Mean \pm SD. & $16.16 \pm 0.63 \mathrm{~mm}$ & $21.46 \pm 0.6 \mathrm{~mm}$ & $32.69 \pm 2.233$ \\
& $16.2 \mathrm{~mm}$ & $21.5 \mathrm{~mm}$ & 32.7 \\
\hline
\end{tabular}

Comparison between immediate post-operative CBCT and delayed (4 months) post-operative CBCT was done to evaluate the vertical bone stability. The gained vertical height remained stable except in case no 1 where $0.2 \mathrm{~mm}$ bone loss had occured.

\section{Bone density evaluation}

The preoperative bone density ranged from $432 \mathrm{HU}$ to $480 \mathrm{HU}$, with a mean of $449.5 \pm 14.32 \mathrm{HU}$.

Immediate post-operative CBCT was done to be compared with the preoperative CBCT to evaluate the bone density after application of the grafted material.

A comparison between immediate post-operative CBCT and delayed (4 months) post-operative CBCT was done to evaluate the actual bone density developed after consolidation of the grafted material.

On the 4th post-operative month there was a statistically significant change in bone density to range from $846 \mathrm{HU}$ to $869 \mathrm{HU}$ with a mean of $(854.8 \pm 6.84 \mathrm{HU})$ and a mean percentage change of $90.28 \%$. (Table 2 )

Table 2: Comparison between preoperative and postoperative bone density.

\begin{tabular}{|l|c|c|c|}
\hline \multirow{2}{*}{} & \multicolumn{3}{|c|}{ Bone density } \\
\cline { 2 - 4 } & Preoperative & $\begin{array}{c}\text { Delayed } \\
\text { post- } \\
\text { operative }\end{array}$ & \% change \\
\hline Min.-Max. & $432-480 \mathrm{HU}$ & $\mathbf{8 4 6 - 8 6 9} \mathrm{HU}$ & $\mathbf{8 1 - 9 5 . 8}$ \\
Mean \pm SD. & $449.5 \pm 14.3 \mathrm{HU}$ & $\begin{array}{c}854.5 \pm 6.8 \\
\text { HU }\end{array}$ & $90.28 \pm 4.535$ \\
& $447.5 \mathrm{HU}$ & $853.5 \mathrm{HU}$ & 90.15 \\
\hline Median & & & \\
& & & \\
\hline
\end{tabular}




\section{DISCUSSION}

The present study was conducted on eight patients from both genders, with age ranging between 55-64 years. The patients were selected from the outpatient clinic of the Oral and Maxillofacial Surgery Department, and the removable Prosthodontic Department at the Faculty of Dentistry, Alexandria University.

One of the key factors for the success of bone grafting surgery is appropriate patient selection, which indicates a thorough medical history that should be carefully registered to exclude any systemic disease that might have an adverse effect on bone healing process (11). Also assessment of patients' oral hygiene and condition of oral mucosa covering the deficient ridge were done to ensure healthy tissues and exclude inflamed mucosa that might retard healing \& avoid direct damaging effect of bacterial and inflammatory by- products (12).

In this study all patients were instructed to follow strict oral hygiene measures by using Chlorhexidine $0.12 \%$ mouth wash at least one week before the surgery. Also absence of inflammation in the overlying mucosa was checked prior to surgery.

This study targeted mainly non-smoker patients as smoking is known to have an association to the failure of graft surgeries (13). Levin \& Schwartz-Arad in 2005 (14) stated that Cigarette smoking may adversely affect wound healing, and thus, jeopardize the success of bone grafting. Their study also described how tobacco directly affects osteoblast function and how smoking by-products such as carbon monoxide and cyanide delay wound healing together with nicotine which inhibit cell proliferation and so they are considered as risk factors for impaired healing.

Herzberg, Dolev \& Schwartz-Arad in 2006 (15) also stated that one possible mechanism by which smoking might affect bone formation is lowering blood flow rate due to increased peripheral resistance and platelet aggregation.

In this study a labial mucoperiosteal flap was reflected without the involvement of crestal or lingual periosteum to ensure copious blood supply. Blood supply for the osteomatized segment should be sufficient to avoid further complications. As some complications might occur like osteomyelitis, lack of bone formation, and necrosis of the superior segment because of the resorbed mandible with its poor blood supply (16).

To obtain a safe cutting of the osteomatized segment without damaging the adjacent soft tissues and preservation of lingual periosteum, a piezoelectric device was used. It operates at a modulated frequency of $25-35 \mathrm{kHz}$, producing micro-vibration of 60 to $200 \mathrm{~mm} / \mathrm{s}$ that cuts only and precisely in mineralized tissues unlike conventional methods of bone cutting. This yielded a preserved blood supply for the osteomatized segments in all patients and none of the previously discussed complications have occurred.

Jee-Won Moon in 2009 (17) in his study mentioned that the use of piezoelectric device minimized the risk of harming the lingual flap and periosteum with minimal loss of bone. The accuracy and selectivity of the piezoelectric device make it superior to conventional saw or bur.

Also, Dong-Seok Sohn in 2010 (16) stated that the piezoelectric bone surgery is beneficial for the sandwich augmentation procedure as the lingual periosteum and flap can be maintained for maximum blood supply because of the precise bony cutting without soft tissue involvement.
Moreover, Carini et al in 2014 (18), reported that bone tissue healing showed a reduced rate of bone loss with piezoelectric instruments than with conventional devices, as well as a better healing by reducing patients' post- operative morbidity. Peker and Tekdal et al in 2105 (19) also confirmed that piezoelectric surgery offers a less traumatic osteotomy modality than drilling.

Synthetic HA and ß-TCP was used in this study as a mineralized allograft material that was inserted between the basal bone and the oteomatized segment. In all cases favorable new bone formation was shown radiographically 4-6 months post-operatively.

A study done by Gupta in 2016 (20) concluded that TCP/HA mixtures stimulated the differentiation of bone marrow stromal cells toward osteoblastic cells, and therefore may be beneficial in gradient microsphere-based scaffolds for osteochondral regeneration.

In the present study autologous Injectable-PRF (I-PRF) was injected on the synthetic hydroxy apatite (HA) betatricalcium phosphate (ß-TCP) particles to form a "bone graft steak" and minimize the spreading and migration of the bone graft particles away from the site of augmentation.

In 2015 Mourão et al (21) stated that the use of I-PRF in combination with graft material can produce a bone graft steak.The possibility of bonding of i-PRF with biomaterials for bone grafting creates an alternative to PRP as a platelet aggregate for bone regeneration.

In 2017, Miron et al (22), performed a study to compare injectable-PRF (i-PRF) with Platelet rich plasma (PRP) that uses anticoagulant agents in an attempt to overcome the concerns that have been expressed regarding the use of anticoagulant agents known to inhibit wound healing.They demonstrated the ability of I-PRF to release higher concentrations of various growth factors and induced higher fibroblast migration and expression of PDGF, TGF- $\beta$, and collagen1. Also, a potent formulation of liquid platelet concentrates could be obtained without the use of anticoagulants.

In this study, a PRF membrane was used as a protective, mechanical barrier upon the bone graft steak. Choukroun in 2006 (9) stated that the addition of PRF to the bone graft can lead to a reduction of the volume of bone substitutes used and seems to improve revascularization of the graft by supporting angiogenesis.

Moreover, Toffler in 2009 (23) and Delcorso in 2010 (24) stated that PRF membranes help in wound healing, protecting the surgical site, promoting soft tissue repair; when mixed with bone graft, it may act as a "biological connector”, which attracts stem cell, favors the migration of osteoprogenitor cells to the center of the graft, and provides a neo-angiogenesis.

Also, Kawase et al in 2015 (25) stated that PRF membrane is well cross-linked and exhibits adequate stiffness to serve as a barrier membrane.

Fixation by titanium micro-plates and screws was done prior to insertion of the grafted material in our study. That was done in an attempt to increase the crestal bone stability and reduce long term bone resorption.

This is in accordance, with Jensen in 2006 (26) who published a retrospective study to evaluate the crestal stability of alveolar augmentation using an autogenous interpositional bone graft and the use of bone plates for fixation .Eight patients with 10 graft sites were followed from 1 to 4 years with panoramic evaluation to determine if 
dimension changes of the alveolar graft sites had occurred. The crestal height was maintained with no bone resorption in 8 patients during the follow up period

Clinically healing was uneventful in all cases included in this study with the absence of postoperative complications except for one case who exhibited wound dehiscence in some areas along the incision line and this case was treated by daily local irrigation with saline mixed with broad spectrum antibiotic cefotaxime $1 \mathrm{~g}$ vial (Cefotax, EPICO, Egypt) on dental chair and at the same time the patient was instructed to maintain meticulous oral hygiene using Chlorhexidine and warm saline mouthwash at home. The dehiscence healed spontaneously 3 weeks later with the exfoliation of small bony spicule. That was believed due to tension created in the flap during its closure. This is in agreement with Laviv et al 2014 (27), where their study evaluated alveolar sandwich osteotomy technique in resorbed alveolar ridge at 10 grafted sites ( 5 maxillary and 5 mandibular) in 9 patients, and they have stated that 2 mandibular cases have experienced wound dehiscence.

Also, patients had some transient postsurgical paresthesia, that lasted for maximum 6 weeks postoperatively and recovered without any medications. And that was due to retraction of the flap. This is in accordance with Jensen in 2006 (26) who stated that transient paresthesia was present in all patients included in his study and it was due to flap retraction of the mental nerve.

In the present study, a post-operative CBCT 4 months later showed a significant increase in bone density This result demonstrate a change in bone density from the less favorable type D3 preoperatively to more favorable type D2 postoperatively according to Misch bone density classification. Nilüfer Bölükbaşı et al in 2013 (8) concluded that PRF in addition to BCP may favor the formation of new bone.

In addition, comparing preoperative and postoperative CBCT showed a significant increase in bone height. The total overall bone height at the end of the procedure at 4 months ranged from $5 \mathrm{~mm}$ to $5.6 \mathrm{~mm}$ with a mean $5.3 \pm$ $0.2 \mathrm{~mm}$. When comparing the delayed (4 months) postoperative with the immediate post-operative CBCT the vertical height was the same without any change except in case no 1 where $0.2 \mathrm{~mm}$ bone loss have occured.

This is in agreement with Jee-Won Moon et al in 2009 (17) where they were evaluating reconstruction of atrophic anterior mandible by sandwich osteotomy technique using piezoelectric device, titanium plates and screws and allograft as inter-positional material. They yielded similar results as the alveolar bone height was increased up to about $10 \mathrm{~mm}$ and the study verified that the initial bone gain underwent minimal bone resorption, indicating favorable stability of the augmented bone over time.

Also, Dong-Seok Sohn et al in 2010 (16) who was evaluating sandwich osteotomy technique in posterior mandibles of 3 patients, where allograft inter-postional material was used and fixation with titanium plates and screws was done in 2 patients only. Their study resulted in $6 \mathrm{~mm}$ bone gain and the segmented bone showed less bone resorption in the fixation cases than in non-fixation case.

Moreover, Laviv et al in 2014 (27), mentioned in their study that there was $4-10 \mathrm{~mm}$ (mean 6 ) bone gain which remained stable after 4-6 months post operatively.

On the other hand, Choi et al in 2004 (28) studied the sandwich osteotomy with interpositional allograft in the atrophic anterior mandible of 9 patients without fixation. Their study reported vertical bone augmentation ranging from 8.4 to $11.0 \mathrm{~mm}$ (mean $9.8 \mathrm{~mm}$ ), and some resorption in crestal bone height of $18 \%$ to $28 \%$ after 3 months.

Sandwich osteotomy technique using piezoelectric device, IPRF mixed with HA and B-TCP as a graft material with fixation of the segmented bone with titanium plates and screws is a reliable method for augmenting vertically resorbed mandibles and could yield satisfactory vertical bone gain with minimal resorption.

\section{CONCLUSION}

From the results of this study, it can be concluded that sandwich osteotomy technique using piezoelectric device and a mixture of IPRF \& Ha-BTCP is a reliable bone augmentation method that can restore deficient vertical bone height and the newly formed bone showed with minimal complications.

\section{CONFLICT OF INTEREST}

The authors declare that they have no conflicts of interest.

\section{REFERENCES}

1. Mercier P, Lafontant R. Residual alveolar ridge atrophy: classification and influence of facial morphology. J Prosthet Dent. 1979; 41: 90-100.

2. Esposito M, Grusovin MG, Felice P, Karatzopoulos G, Worthington HV, Coulthard P. Interventions for replacing missing teeth: horizontal and vertical bone augmentation techniques for dental implant treatment. Cochrane Database Syst Rev. 2009; 7:CD003607.

3. Bormann KH, Suarez-Cunqueiro MM, von See C, Kokemuller H, Schumann P, Gellrich NC. Sandwich osteotomy for vertical and transversal augmentation of the posterior mandible. Int J Oral Maxillofac Surg. 2010; 39: 554-60.

4. Schettler D, Holtermann W. Clinical and experimental results of a sandwich-technique for mandibular alveolar ridge augmentation. J Maxillofac Surg. 1977; 5: 199-202.

5. Luigi L, Giovanna I, Adriano P, Lorenzo, and Marco C. Vertical ridge augmentation of the atrophic posterior mandible with sandwich technique: Bone block from the chin area versus corticocancellous bone block allograftclinical and histological prospective randomized controlled study. Biomed Res Int. 2014; 2014: 982104.

6. Strietzel FP, Reichart PA, Graf HL. Lateral alveolar ridge augmentation using a synthetic nano-crystalline hydroxyapatite bone substitution material (Ostim): preliminary clinical and histological results. Clin Oral Implants Res. 2007; 18: 743-51.

7. Saikia KC, Bhattacharya TD, Bhuyan SK, Talukdar DJ, Saikia SP, P Jitesh. Calcium phosphate ceramics as bone graft substitutes in filling bone tumor defects. Indian J Orthop. 2008; 42: 169-72.

8. Nilüfer B, Sinem Y, Merva S T, Kemal A. The use of platelet-rich fibrin in combination with biphasic calcium phosphate in the treatment of bone defects: a histologic and histomorphometric study. Curr Ther Res Clin Exp. 2013; 75: 15-21. 
9. Choukran J, Diss A, Simonpieri A, Girard MO, Schoeffler C, Dohan SL, et al. Platelet Rich Fibrin (PRF): A second generation platelet concentrate. Part IV: Clinical effects on tissue healing. Oral Surg Oral Med Pathol Oral Radiol Endod. 2006; 101: 56-60.

10. Jensen MP, Karoly P, Braver S. The measurement of clinical pain intensity: a comparison of six methods. Pain. 1986; 27: 117-26.

11. Moy PK, Medina D, Shetty V, Aghaloo TL. Dental implant failure rates and associated risk factors.Int J Oral Maxillofac Implants. 2005; 20: 569-77.

12. Keller EE, Tolman DE, Eckert SE. Maxillary antral-nasal inlay autogenous bone graft reconstruction of compromised maxilla: a 12-year retrospective study. Int J Oral Maxillofac Implants. 1999; 14: 707-21.

13. Blomqvist JE, Alberius P, Isaksson S, Linde A, Hansson BG. Factors in implant integration failure after bone grafting: an osteometric and endocrinologic matched analysis. Int J Oral Maxillofac Surg. 1996; 25: 63-8.

14. Levin L, Schwartz-Arad D. The effect of cigarette smoking on dental implants and related surgery. Implant Dent. 2005; 14: 357-61.

15. Herzberg R, Dolev E, Schwartz-Arad D. Implant marginal bone loss in maxillary sinus graft. Int $\mathrm{J}$ Oral Maxillofac Implants.2005; 21: 103-10.

16. Sohn DS, Shin HI, Ahn MR, Lee JS. Piezoelectric vertical bone augmentation using the sandwich technique in an atrophic mandible and histomorphometric analysis of mineral allografts: a case report series. Int J Periodontics Restorative Dent. 2010; 30: 383-91.

17. Jee-Won M, Byung-J, Won-Hyuk L, Kyung M, Sohn DS. Reconstruction of Atrophic Anterior Mandible Using Piezoelectric Sandwich Osteotomy: A Case Report. Implant Dent 2009; 18: 195-202.

18. Carini F, Saggese V, Porcaro G, Baldoni M. Piezoelectric surgery in dentistry: a review. Minerva Stomatol 2014; 63: 7-34.

19. Peker Tekdal G, Bostanci N, Belibasakis GN, Gurkan A. The effect of piezoelectric surgery implant osteotomyon radiological and molecular parameters of peri-implant crestal bone loss: a randomized, controlled, split-mouth trial. Clin Oral Implants Res 2015; 16: 1-10.

20. Gupta V, Dina VL, Barragan M, Berkland CJ, Detamore MS. Microsphere-based scaffolds encapsulating tricalcium phosphate and hydroxyapatite for bone regeneration. J Mater Sci Mater Med 2016; 27: 121.

21. Mourão CF, Valiense H, Melo ER, Mourão NB, Maia MD. Obtention of injectable platelets rich-fibrin (i-PRF) and its polymerization with bone graft: technical note. Rev Col Bras Cir. 2015; 42: 421-3.

22. Miron RJ, Hernandez M, Kandalam U, Zhang Y, Ghanaati S, Choukroun J et al. Injectable platelet rich fibrin (i-PRF): opportunities in regenerative dentistry? Clin Oral Investig. 2017.

23. Toffler M, Toscano N, Holtzclaw D, Corso MD, Dohan Ehrenfest DM. Introducing Choukroun's platelet rich fibrin (PRF) to the reconstructive surgery milieu. J Implant Clin Adv Dent. 2009; 1: 21-30.

24. Del Corso M, Toffler M, Dohan Ehrenfest DM. Use of an autologous leukocyte and platelet-rich fibrin (L-PRF) membrane in post-avulsion sites: an overview of Choukroun's PRF. J Implant Adv Clin Dent. 2010; 1: 2735.

25. Kawase T, Kamiya M, Kobayashi M, Tanaka T, Okuda $\mathrm{K}$, Wolff, LF, et al. The heat-compressiontechnique for the conversion of platelet-rich fibrin preparation to a barrier membrane with a reduced rate ofbiodegradation. J Biomed Mater Res B Appl Biomater. 2015; 103: 82531.

26. Jensen OT, Kuhlke L, Bedard JF. Alveolar segmental sandwich osteotomy for anterior maxillary vertical augmentation prior to implant placement. J Oral Maxillofac Surg. 2006; 64: 290-6.

27. Amir L, Ole TJ, Eyal T, Nardy C. Alveolar Sandwich Osteotomy in resorbed alveolar ridge for dental implants: A 4 years prospective study. J Oral and Maxillofac Surg. 2014; 72: 292-303.

28. Choi BH, Lee SH, Young JH, Gyun Han S. Use of the sandwich osteotomy plus an interpositional allograft for vertical augmentation of the alveolar ridge. J CranioMaxillofac Surg 2004; 32: 51-4. 